\title{
Aporte sedimentario, morfologías eólicas y procesos de formación del registro arqueológico en campos de dunas del golfo San Matías (Río Negro, Argentina)
}

Sedimentary contributions, wind morphologies and formation processes of the archaeological record in dune fields of the San Matías gulf (Río Negro, Argentina)

RESUMEN

Objetivo. Establecer la relación entre aporte sedimentario, morfologías eólicas y procesos de formación del registro arqueológico en la costa norte del Golfo San Matías (Río Negro). Para dar cuenta de ello, la metodología aplicada consistió en una revisión exhaustiva de la literatura geológica, geomorfológica y geoarqueológica, así como visitas a campo, en las que se recolectaron muestras sedimentarias da varias unidades geomorfológicas, para posteriores análisis granulométricos. Los resultados obtenidos, dan cuenta que, los ambientes litorales, en respuesta a ese dinamismo, de un lado facilitaron la formación de diferentes tipos de morfologías, tales como las dunas, y por el otro, tanto la dinámica costera como la actividad antrópica han afectado las propiedades cualitativas y cuantitativas de la evidencia. Conclusión. Debido a las condiciones ambientales de la región de estudio, el material arqueológico continuamente esté siendo expuesto, redepositado y desplazado por la acción eólica, afectando así el contexto primario de depositación.

Palabras clave: procesos de formación, dunas, registro arqueológico, preservación, geoarqueología.
Dalila Viviana

Herrera-Villegas

Candidata a Doctora

en Arqueología.

UNICEN. INCUAPA - CONICET.

Olavarría, Argentina.

$\square$ dalila.1083@gmail.com

(1) ORCID: 0000-0001-8942-9185

$\checkmark$ Google Scholar 


\begin{abstract}
Objective: To establish the relationship between sedimentary contribution, wind morphologies and processes of formation of the archaeological record on the north coast of the San Matías Gulf (Río Negro).For this purpose, the applied methodology consisted of an exhaustive review of the geological, geomorphological and geoarchaeological literature, as well as field visits, in which sedimentary samples were collected from several geomorphological units for subsequent granulometric analyzes. The results obtained show that coastal environments, in response to this dynamism, on the one hand facilitated the formation of different types of morphologies, such as dunes and, on the other hand, both coastal dynamics and anthropic activity have affected qualitative and quantitative properties of the evidence. Conclusion: due to the environmental conditions of the region studied, the archaeological material is continually being exposed, redeposited and displaced by wind action, thus affecting the primary deposition context.
\end{abstract}

Keywords: formation processes, dunes, archaeological record, preservation, geoarchaeology.

\title{
Introducción
}

os ambientes costeros suelen caracterizarse por ser altamente dinámicos, característica que, en el caso de la costa norte del Golfo de San Matías ha dado paso a la formación de diferentes tipos de dunas (parabólicas y barjanoides) (Figuras 2 y 3), las mismas que, además de tener su propia dinámica, son las unidades sobre las que se encuentran emplazados los sitios arqueológicos, los cuales están conformados por una serie de concheros que se encuentran ubicados a lo largo de esta costa. Debido al estado de preservación en el que se encuentra la evidencia, es que las dunas, espacialmente las barjanoides son consideradas hasta el momento las principales unidades portadoras del registro arqueológico (Favier-Dubois et al., 2016), ya que por su alta dinámica de depositación sedimentaria ha permitido que muchas de las propiedades del registro aún se conserven. En este sentido, se propone como objetivo establecer la relación entre aporte sedimentario, morfologías eólicas y procesos de formación del registro arqueológicos en dicha costa.

En este marco se buscó analizar desde la geoarqueología varios aspectos, uno, cómo la dinámica costera a través de factores como viento, precipitación, humedad, vegetación y sedimentos disponibles, ha influido 
en la morfología dunar; y dos, como estos agentes más la actividad antrópica ha afectado las condiciones primarias de depositación, preservación y disposición actual del registro arqueológico en el tiempo. De esta manera, a partir de la literatura disponible sobre ambientes costeros y morfologías eólicas se buscó darles sustento a las muestras de sedimentos recolectadas de la playa actual y diferentes puntos de la terraza holocena. El fundamento de esta recolección consistió, en llevar a cabo un análisis comparativo de los sedimentos recolectados en cada punto establecido, con el fin último de establecer si la relación entre playa y duna sigue siendo representativa, ya que esta podría estar favoreciendo de un lado la preservación a largo plazo de los concheros que llevan poco tiempo en exposición, y del otro permitir que los que llevan más tiempo de exposición puedan conservar parte de su historia de formación. De acuerdo con esto, se espera entonces dar cuenta de las características contextuales que actualmente presenta el registro arqueológico y de los factores que han intervenido en las propiedades de los depósitos presentes en la costa norte del golfo San Matías.

\section{Materiales y métodos}

La metodología aplicada en este trabajo consistió en realizar un acercamiento geoarqueológico a la zona de estudio la cual se realizó en varias etapas, la primera tuvo que ver con una revisión exhaustiva de la literatura costera, arqueológica, geológica y paleoambiental. Con base en esta información, se definieron los principales tipos de costa y su relación con la disponibilidad de recursos (Favier Dubois y Borella, 2011). De otro lado, se pudo dar cuenta que el desarrollo morfológico de las dunas se debe a variaciones del nivel del mar durante el Holoceno (Schellmann y Radtke, 2010). Lo que es sustentado por trabajos paleoclimáticos previamente realizados en la costa nortpatagónica (Schäbitz, 2003). Con estos datos fue posible establecer la relación de estas variables con la preservación, visibilidad y disposición actual de la evidencia arqueológica.

Para tener una mayor aproximación al proceso sedimentario en la localidad de Bahía Creek, principal campo de dunas en toda la costa norte de Patagonia, se llevó a cabo la recolección de muestras sedimentarias, para lo cual se determinó realizar una transecta de muestreo que abarcara diferentes unidades geomorfológicas que incluían la playa actual, terraza holocena, dunas y conchero sobre duna (Figura 5). Con estas muestras se buscaba realizar un análisis granulométrico que permitiera determinar el origen y dinámica sedimentaria entre las dunas y la playa, así como la relación playa-duna. Asimismo, es posible establecer las probabilidades 
de que la duna conserve su morfología y que el registro arqueológico preserve sus propiedades de formación, ya que muchas de las características de la evidencia cultural están directamente relacionadas con la dinámica costera, específicamente con la sedimentaria. Para la determinación de la estructura de los granos y el predominio mineralógico de cada muestra se utilizaron $10 \mathrm{~g}$ de sedimentos que fueron analizados en una lupa estereoscópica marca Hokenn optik modelo ZTX E ZOOM con un aumento de 10x.

Los estudios granulométricos se realizaron utilizando la clasificación USA standard ASTM E 11-61, para el peso de las muestras se utilizó una balanza electrónica (A\&D FX 3000i). El proceso de tamizaje se realizó en varias etapas. La primera tuvo que ver con la selección de abertura de los tamices, posteriormente, las muestras se procesaron en los tamices hasta lograr retener el tamaño de grano correspondiente para cada tamaño granulométrico. Después se creó una tabla de Excel en la que se ingresó el peso retenido de cada muestra. Con estos datos se creó un histograma en el que fuera posible dar cuenta del porcentaje en peso de cada muestra recolectada de las unidades geomorfológicas previamente citadas. Finalmente, con estos estos análisis fue posible dar cuenta de la estrecha relación que sigue existiendo entre la playa y duna y de la importancia que esta tiene no solo en la morfología de estos cuerpos eólicos sino en la preservación de las propiedades contextuales del registro arqueológico.

\section{Área de estudio}

La zona de estudio se extiende desde el Balneario el Cóndor hasta la Bahía de San Antonio, litoral que posee una dirección general Este-Oeste (Figura 1), caracterizándose por un clima templado semiárido, con temperaturas de unos $12^{\circ} \mathrm{C}$ y precipitaciones medias entre 100 y $350 \mathrm{~mm}$ anuales (Labraga y Villalba 2009). Las mareas presentan un régimen semidiurno y una amplitud media de 3,2 $\mathrm{m}$ en la desembocadura del río Negro, mientras que a la entrada de la bahía de San Antonio ésta es de 7,1 m (Capítoli, 1973). Los vientos predominantes soplan del cuadrante oeste (NO, O y SO), presentando mayor intensidad entre los meses de septiembre y enero, alcanzando velocidades históricas máximas las del cuadrante SO (Crisoliti y Pahissa-Campá, 1973). En cuanto a la vegetación, domina la estepa arbustiva correspondiente a la provincia de monte, distrito sur (Cabrera y Willink, 1980; Roig et al., 2009) predominando el tomillo, la jarilla y el molle, mientras que en algunos sectores se observa el algarrobo patagónico y algo de chañar (Bran et al., 1985). 


\section{Antecedentes geológicos y paleoambientales}

La costa rionegrina presenta una variedad de condiciones geológicas y geomorfológicas particulares y una oferta ambiental heterogénea. En este sentido la costa presenta la alternancia de elevados acantilados y depresiones topográficas que constituyen entrantes costeros donde se desarrollan playas arenosas. Los acantilados poseen hasta $50 \mathrm{~m}$ y $60 \mathrm{~m}$ de altura y presentan plataformas litorales cuya extensión varía entre 150 y $200 \mathrm{~m}$, lo que hace posible encontrar una variedad de especies potencialmente explotables como moluscos sésiles (fijos a las rocas) en el intermareal medio e inferior, cholgas, mejillones, mejillín y abundancia de cangrejo rojo bajo las rocas (Favier Dubois y Borella, 2011).

En ocasiones los bivalvos mencionados presentan una densidad mayor en sectores lindantes con playas arenosas, como Punta Mejillón y Bahía Creek (figura 1). En las playas y marismas arenosas que alternan con las plataformas rocosas se puede encontrar almeja blanca y púrpura y gasterópodos como bocinas y trofones. Para la Caleta de los Loros se reportan mejillones y mejillines adheridos a raíces y tallos en el espartillar, el cual también se desarrolla en la bahía de San Antonio donde alberga comunidades de mejillines (Favier Dubois y Borella, 2011). Los entrantes costeros que alternan con los acantilados corresponden a bajos topográficos, tales como el bajo vinculado al Bajo de la Quinta, a Caleta de los Loros-Bahía Creek y el Bajo de San Antonio (figura 1), los cuales debido a su morfología no solo se constituyeron en vías privilegiadas de acceso al mar, sino que facilitaron el desarrollo de cordones de gravas, playas arenosas y campos de dunas, desatancándose entre ellos el que es considerado el mayor campo de dunas de la zona, que va desde la Caleta de los Loros hasta Bahía Creek, con dirección SO-NE (la de los vientos más intensos) introduciéndose $45 \mathrm{~km}$ tierra adentro (Favier Dubois y Borella, 2011, Fig. 1).

Como se mencionó, en esta franja costera se reportan grupos morfológicos de dunas con cronologías disímiles, que son principalmente dos: parabólicas y barjanoides. Estas son producto de variaciones en la disponibilidad de arena en la playa en función de cambios del nivel del mar. Los estudios geológicos y geomorfológicos realizados en las proximidades de la región dan cuenta de condiciones áridas durante el máximo transgresivo del Holoceno medio (ca. 7400 años AP), periodo en el que las elevadas temperaturas globales provocaron que el nivel del mar aumentara entre 6 y $7 \mathrm{~m}$ por encima del nivel actual. Posteriormente, hacia el $6000 \mathrm{AP}$ se produce un primer pulso de descenso del nivel marino en la costa patagónica (Schellmann y Radtke, 2010) al que le sigue una etapa de estabilidad 
hasta hace unos 3000-2600 años AP. Durante esta etapa, cuando el aporte sedimentario de la playa no es abundante debido al alto nivel del mar, se empiezan a desarrollar lo que hoy se observa como campos de dunas parabólicas en la región (Figura 2). Luego, entre ca. 2600-2400 años AP se reporta un segundo pulso de descenso del nivel del mar no menor a 1 m (Schellmann y Radtke, 2010), que favoreció un mayor desarrollo de cuerpos eólicos en forma de grandes campos de dunas barjanoides, particularidad relacionada directamente con una mayor provisión y movilidad de arena, condición que ha valido para que estas dunas sean consideradas las más abundantes en toda la costa norte (Figura 2).

En esta región el escenario paleoclimático del Holoceno medio se corresponde con las condiciones más áridas para el Holoceno en la costa norte patagónica (Schäbitz, 1994, 2003; Favier Dubois et al., 2016). A este respecto el principal antecedente paleoclimático lo constituyen los estudios polínicos realizados por Schäbitz $(1994,2003)$ en lagunas secas del nordeste patagónico, que indican condiciones áridas durante el Holoceno medio (ca. 7500 años 14C AP) con elevadas temperaturas y bajas precipitaciones. Estas condiciones áridas cambiaron levemente hacia semiáridas entre ca. 5000 años $14 \mathrm{C}$ AP y ca. 3000 años $14 \mathrm{C}$ AP, hasta que, de acuerdo con este autor, en ca. 3000-2500 años 14C AP, se establecieron las condiciones semiáridas actuales más benignas, con el incremento de precipitaciones anuales (Schäbitz 2003).

De la misma manera, los estudios paleoambientales realizados sobre polen obtenido de dos testigos en la laguna temporaria Bajo de la Quinta señalan entre 6800-3000 años 14C AP la presencia de una estepa arbustiva xerofítica bajo condiciones climáticas predominantemente áridas, y luego un cambio a semiáridas hacia el Holoceno tardío (ca. 3000 años 14C AP), lo que correspondería con el esquema propuesto por Schäbitz (Favier Dubois et al., 2016). No obstante, alrededor de los 1000 años $14 \mathrm{C}$ AP, se registra un nuevo pulso árido inferido por la reaparición de tipos polínicos similares a los del primer bloque temporal (Marcos et al., 2012). Este pulso podría representar la expresión en la costa de anomalías de precipitación y temperatura referibles al período cálido medieval, detectadas previamente por estudios dendroclimatológicos en el NO de Patagonia (Villalba, 1994).

\section{Resultados}

Para entender el comportamiento actual del registro arqueológico y su historia de formación es fundamental conocer las características geológicas y geomorfológicas de cada zona de estudio, ya que esto 
permite adquirir un panorama amplio de cómo y por qué los paisajes cambian, pero sobre todo de cómo los factores externos que modificaron el paisaje pueden intervenir en las condiciones contextuales de los depósitos culturales. En este sentido, se destacarán las principales variables que han contribuido, por un lado, a la formación de las dunas y, por el otro, a la disposición actual de la evidencia, permitiendo de este modo generar un panorama interpretativo menos complejo, principalmente cuando se trata de dar cuenta de los procesos de formación de los sitios arqueológicos.

\section{Procesos de formación del registro arqueológico en Bahía Creek}

\section{Desarrollo dunar}

En la evolución de una duna costera deben conjugarse una serie de factores que permitan su desarrollo y morfología característica. En el caso específico de la costa norte del Golfo de San Matías, especialmente en Bahía Creek, principal campo de dunas, es común encontrar morfologías tipo barjanoides (Figura 2), cuya particularidad está relacionada directamente con una mayor provisión y movilidad de arena, producto de cambios del nivel del mar ocurridos durante el Holoceno tardío (Favier-Dubois et al., 2016). Para que estos cuerpos eólicos lleguen a adquirir su morfología característica deben darse una serie de factores como: suministro constante de material sedimentario, sea por deriva litoral o corrientes marinas (Sanjaume et al., 2011), fuertes vientos que provengan desde el mar hacia el interior del continente, escasa humedad atmosférica y presencia de vegetación (McKee, 1983).

Por otra parte, la capacidad que pueda llegar a tener el viento para remover, transportar y sedimentar, son aspectos que van a contribuir al desarrollo de la duna. Dicho lo anterior, la remoción hace referencia a la capacidad del viento para levantar partículas de arena (Warren, 1979), las cuales son lanzadas a las corrientes de aire que, una vez removidas, empiezan a moverse por diferentes mecanismos como suspensión, saltación y reptación (Sanjaume et al., 2011) (Figura 3). Una vez que las partículas son removidas, el transporte que realiza el viento hace que la tasa de transferencia varíe con relación a la velocidad de este. Luego, la sedimentación es la encargada del equilibrio y morfología de la duna, dado que existe una relación directa entre las corrientes de aire y la génesis de la duna, pues la sedimentación se produce cuando el aire está saturado de arena (Sanjaume et al., 2011) que, al chocar con un obstáculo como la vegetación, hace que la intensidad de este disminuya quedando el material arenoso atrapado en la superficie del suelo 
(Ley et al., 2007). Hay que mencionar, además, que cuando hay variación en las líneas de corriente por cambios en la velocidad del viento, es cuando las dunas adoptan diferentes formas (Goldsmith, 1985).

\section{El rol de la vegetación en las dunas}

La vegetación juega un papel vital en el crecimiento de las dunas (Klijin, 1990), pues le introduce rugosidad a la superficie haciendo que la velocidad del viento y de transporte disminuya, generando de esta manera una superficie blanda que absorbe gran cantidad de energía producto de los granos de arena que llegan por saltación (Sanjaume et al., 2011). En el caso de las dunas costeras, la vegetación que allí se desarrolle se les conoce con el nombre de Psammófitas y Xerófitas, las cuales se caracterizan por su resistencia al calor, limitación de nutrientes y tolerancia al enterramiento, siendo esta última la que brinda la capacidad de establecerse sobre restos de plantas, aumentando las probabilidades de que la colonización de dichas plantas sea rápida y efectiva, favoreciendo a su vez el desarrollo dunar (Sanjaume et al., 2011). Además, este tipo de planta determinará la capacidad para retener sedimentos, así como el desarrollo y la morfología de la estructura sedimentaria (Ley et al., 2007). Por otra parte, cuando la vegetación empieza a desaparecer por la erosión del viento, deja al descubierto las raíces haciendo que las plantas puedan morir (Tsoar, 1990; Arens, 1996), dejando de ser una barrera eólica, por lo que la duna al quedar desprotegida va generando un paulatino avance tierra adentro, trayendo implicaciones negativas, tanto para los depósitos arqueológicos como para las propiedades cualitativas y cuantitativas de los materiales que los componen.

\section{Resultados granulométricos}

Para entender los procesos actuantes en la localidad objeto de estudio, fue fundamental conocer la dinámica sedimentaria, para poder dar cuenta tanto de las propiedades actuales del contexto de hallazgo de los depósitos, como de los materiales que los conforman.

En este sentido, se detallan a continuación algunos de los resultados producto del análisis granulométrico realizado al material sedimentológico de las diferentes unidades geomorfológicas: playa actual, terraza holocena, duna sobre terraza holocena y conchero sobre duna (Figura 4). En términos mineralógicos, la muestra arrojó un dominio de cuarzos y feldespatos, en cuanto a la estructura, esta presentó granos entre subangulares y sub-redondeados. Por su parte, la distribución de las muestras evidencia un dominio que osciló entre finas y muy finas 
(Figura 5), lo que sería correspondiente con su origen eólico, es decir, con la capacidad que tiene el viento para remover, transportar y sedimentar, característica que favoreció el sepultamiento rápido y la preservación de los depósitos arqueológicos que quedaron incluidos en ellas a lo largo del tiempo. Posteriormente, de acuerdo con el registro se deduce que la vegetación fue estabilizando los cuerpos eólicos que fueron quedando más alejados de las áreas de aporte, a medida que la costa de la bahía se rellenaba por cordones litorales y progradaba.

En vista de los resultados de los análisis, se puede decir que, debido a los constantes y fuertes vientos del cuadrante oeste, la dinámica sedimentaria entre la playa y la duna sigue siendo altamente positiva, ya que, de un lado, abre las probabilidades de que la morfología y movilidad de las dunas se mantenga activa, y del otro, que los depósitos que llevan poco tiempo de exposición puedan tener una continuidad en el tiempo.

\section{Contexto actual del registro arqueológico en Bahía Creek}

A lo largo de este artículo se destacó que los yacimientos arqueológicos se ven afectados diferencialmente de acuerdo con el contexto medioambiental en el que se están emplazados. Con base en esto, la propuesta teórico-metodológica deberá ajustarse y responder en la medida de lo posible a las problemáticas propias de cada ambiente. Por lo tanto el investigador, en un contexto tan dinámico como el costero, tendrá que considerar cada variable que participa en la configuración actual del registro arqueológico

En este sentido, es importante resaltar que los procesos de formación de sitios arqueológicos tienen que ver con aspectos tanto de tipo cultural como natural que han actuado y actúan sobre el registro, y que suelen determinar las características espacio-temporales de la evidencia y su estado de preservación. En este punto, Schiffer (1983) hace hincapié en que es fundamental tener en cuenta la morfología del sitio, el contexto geomorfológico, el sedimento que contiene al registro arqueológico y las transformaciones postdepositacionales para poder entender el comportamiento de la evidencia cultural. De este modo, Butzer (1982) señala que, después que un sitio es abandonado, el registro arqueológico es susceptible de que sus propiedades cualitativas y cuantitativas primarias sean afectadas en mayor o menor medida, especialmente si se tiene en cuenta el dinamismo que estos ambientes tiene para sepultar, erosionar, dispersar y mezclar materiales, entre otros aspectos. 


\section{Impacto ambiental y antrópico}

De acuerdo con lo anterior, queda claro que cada ambiente tiene sus propias particularidades, las mismas que de alguna manera van a tener una participación, directa o indirecta, sobre las propiedades contextuales de los depósitos arqueológicos. Al respecto, Waters y Kuehn (1996) consideran que los paisajes cambian, es decir, nunca son estáticos, sino dinámicos. Acorde con esto, los paisajes costeros no solo tienden a generar cambios en su morfología inicial, sino que suelen dar paso a cambios substanciales de los contextos culturales a lo largo del tiempo, pues al ser ambientes erosivos hacen que cualquier evidencia arqueológica desaparezca o, en el menor de los casos, sea alterada de su contexto original de depositación, dificultando la evaluación o determinación de los usos del espacio o posibles cambios en relación con estos.

En la localidad de Bahía Creek es común encontrar depósitos arqueológicos con diferentes grados de erosión vinculados a dos tipos de afectaciones. El primero, obedece a cambios propios del ambiente, tales como variaciones por sequías cortas o periodos más largos de aridez (McKee, 1983; Waters, 1992). Estos hacen que, por una parte, la duna pierda la vegetación que le brinda estabilidad y cohesión, y por la otra, que los concheros ubicados en dicha zona sean erosionados y expuestos paulatinamente, dando como resultado la presencia de artefactos líticos, óseos, tiestos y, en ocasiones, restos humanos dispersos en las superficies de las hoyadas de deflación (Favier Dubois et al., 2006) (Figura 6). El segundo factor está relacionado con la actividad antrópica, la cual tiene sus inicios a partir del siglo XIX con la introducción de ganado ovino y vacuno, siendo esta una de las causas que más contribuyó en la aparición de focos erosivos y modificación de la cubierta vegetal nativa. Así mismo, la aparición de caminatas y de actividades recreativas, específicamente el uso de vehículos 4x4 ha sido otro detonante en la fragmentación del material lítico, desintegración y compactación de las valvas expuestas (Favier Dubois y Borella, 2007).

\section{Modificaciones cualitativas y cuantitativas del registro arqueológico}

Debido a la exposición prolongada de estos depósitos al ambiente externo, se ha vuelto una constante observar en los materiales diferentes signos de alteración. En este caso, las valvas expuestas presentaron signos de meteorización, que no solo han contribuido a un desgaste diferencial, sino a la fragmentación y transporte de estas y otros materiales a distancias variables que, al modificar su peso, tamaño y forma, se vieron fácilmente desplazadas, tanto por gravedad como por la acción 
eólica (Schiffer, 1983) hacia las zonas de interdunas donde el material es mezclado, muy probablemente, con diferentes depósitos en la superficie deflacionada (Schiffer, 1983; Waters, 1992). Estas modificaciones han permitido dar cuenta de la formación de "palimpsestos espaciales" (Bailey, 2007), lo que incluso puede dar paso a lo que he denominado "falsos espacios de ocupación humana" (Figura 7), que no necesariamente van a corresponder con zonas puntuales de usos humanos, aunque probablemente se les asigne dicha categoría si no se tienen presentes las particularidades de cada ambiente de depositación. Hay que mencionar, además, que las relaciones espaciales que puedan existir entre los diferentes elementos que componen el registro arqueológico pueden ser modificados sustancialmente por procesos eólicos superficiales (Butzer, 1982; Waters, 1992; Schiffer, 1987), ya que el registro continuamente está siendo sometido a varios ciclos de erosión, redepositación y desplazamiento por la acción eólica, alterando significativamente el contexto depositacional primario.

Como consecuencia de lo anterior, las relaciones espacio-temporales de los depósitos están siendo afectadas, ya que se viene observando con frecuencia que estos concheros presentan extensiones en planta que, van de los dos a los seis metros (Favier Dubois, 2016). Esto provoca un aumento de la deflación en los sectores periféricos, haciendo que la longitud y el ángulo de la pendiente del talud crezca, y con ello, el arrastre gravitacional de los restos culturales (Quintana, 2001) (Figura 8). Al presentarse este tipo de alteraciones se da paso a la formación de otro tipo de morfología, lo que dificulta en muchos casos poder establecer los límites reales de los concheros (Figura 8). Otra de las afectaciones que fue posible observar son las modificaciones tafonómicas de los materiales (abrasión o corrasión) (Borrazzo, 2006), las cuales pueden llegar a generar interpretaciones erróneas si no se tiene presente el ambiente de formación, ya que estas pueden ser consideradas como un producto de tipo humano.

Para hacer énfasis a lo anterior, Quintana (2001) da a conocer los resultados de un trabajo realizado en un sitio denominado Punta Ñagué, Chile, en el que expresa que, a raíz de la acción mecánica que provoca la arena al ser transportada por el viento, los artefactos líticos presentaron patinas y superficies pulidas con un notorio desgaste de sus aristas. De igual forma en un trabajo llevado a cabo en bahía San Sebastián (Tierra del Fuego, Argentina) se destaca que los materiales líticos recuperados en superficie presentaban un redondeamiento de sus filos, así como ausencia de este, lo cual lo relaciona con una fractura fresca (Borrazzo, 2006). 
Además de los rasgos presentes en los artefactos líticos, están los cambios tafonómicos generados en restos óseos de aves terrestres y mamíferos marinos. Los mismos, al quedar expuestos mucho tiempo a la intemperie, presentaron signos de decoloración, fracturación, fisuramiento y exfoliación. Además de esto, se evidenciaron improntas radiculares que son ocasionadas por la acción bioquímica de las raíces sobre la superficie ósea, aspecto que permite, de un lado, acercarse a la historia tafonómica de los materiales y, del otro, resaltar que estas marcas en algunos casos suelen ser la única evidencia de que las dunas soportaron comunidades vegetales y muy probablemente dieron paso al desarrollo pedogenético. Sin embargo, la presencia de marcas de raíces rizoconcreciones (Figura 8) sobre las superficies deflacionadas también permite dar cuenta de rasgos pedológicos, ya que estas marcas suelen desarrollarse en horizontes subsuperficiales de suelo (usualmente el horizonte $\mathrm{C}$ ), señalando que el horizonte A ha sido erosionado previamente a la exposición del conchero, probablemente como parte del proceso de degradación que han sufrido las dunas en esta localidad en las últimas décadas (Favier Dubois y Borella, 2007).

A pesar de las condiciones de vulnerabilidad en la que se encuentran los concheros, aún es posible observar que muchos de estos depósitos presentan un alto grado de preservación. Esta cuestión permite sugerir que no permanecieron mucho tiempo expuestos a la intemperie, característica que apunta a un aporte constante de material sedimentario que favoreció un sepultamiento rápido de los concheros y, por ende, una elevada resolución e integridad. Cabe destacar que por resolución se entiende la cantidad de eventos representados en un depósito, y por integridad el número de agentes naturales y antrópicos que intervienen en el proceso de formación de ese registro arqueológico (Binford, 1981). Por consiguiente, se podría considerar que los depósitos arqueológicos forman parte de un patrón estático de asociaciones y covariaciones que, entre cosas, están distribuidas en el espacio (Binford, 1980). Se considera que su composición no necesariamente consta de evidencias de la actividad humana (Binford, 1981) y que independiente del contexto de depositación gran parte del registro arqueológico ha sido formado por los mismos procesos que han modelado el paisaje (Butzer, 1982; Schiffer, 1987; Waters, 1992). 


\section{Consideraciones finales}

Conocer las características depositacionales del registro arqueológico es un factor determinante si lo que se pretende es conocer y entender el comportamiento actual de la evidencia arqueológica. En el caso específico analizado, conocer las particularidades del ambiente costero fue concluyente en la búsqueda por identificar los factores involucrados en el proceso de formación y transformación del registro arqueológico, que incluye los diferentes agentes naturales y culturales que actuaron y actúan sobre los depósitos arqueológicos. Se destaca que estos deben considerarse antes, durante y después del enterramiento de los materiales, pues son estos agentes los que determinan, en gran medida, las interpretaciones que el investigador pueda llegar a hacer del contexto y de las propiedades de los hallazgos.

En este documento el objetivo fue dar cuenta del rol que desempeñan los diferentes agentes naturales y culturales en la configuración y disposición actual de los depósitos. En este sentido, los cambios del nivel del mar ocurridos durante el Holoceno tardío fueron decisivos en el sepultamiento y preservación de los concheros y de sus propiedades, tal como lo da a conocer la conservación diferencial en la que se encuentran muchos de estos. Por otra parte, la actividad antrópica, sin duda, ha sido el agente que más ha afectado el estado de los concheros, ya que, a raíz de la realización de diferentes actividades, muchas de las características de formación de los depósitos han sido alteradas, o en el peor de los casos, destruida en su totalidad. Teniendo en cuenta este panorama, las interpretaciones que se puedan llegar a realizar del contexto actual de la evidencia requiere de un conocimiento de las características ambientales en las que se encuentran emplazados los sitios arqueológicos, ya que sin esta información los resultados que se lleguen a generar estarán cargados de inconsistencias, no solo formacionales sino también relacionados con los usos y función de los espacios.

En cuanto al análisis granulométrico, a través de un estudio comparativo, se evidencia la estrecha relación que sigue existiendo entre la playa y la duna, aspecto que es determinante, tanto en la morfología de la duna, como en la preservación de los concheros que llevan poco tiempo de exposición, permitiendo de este modo conservar a más largo plazo sus características de formación. Finalmente, se cree que, de un lado, fue la continuidad en el aporte sedimentario lo que favoreció que muchos de estos concheros se encuentren en buenas condiciones de conservación y, por el otro, que la dinámica eólica ha sido un aspecto fundamental en la exposición, sepultamiento y salvaguarda de la evidencia arqueológica y sus propiedades. 


\section{Agradecimientos}

El financiamiento para este proyecto fue proporcionado por una subvención de la Agencia Nacional de Promoción Científica y Tecnológica PICT-1128. El autor desea agradecer al Dr. Cristian Favier Dubois por su apoyo y valiosas sugerencias. A la Facultad de Ingeniería UNICEN, por el suministro de espacios y equipamiento.

\section{Referencias}

Arens, S. (1996). Patterns of sand transport on vegetated foredunes. Geomorphology, 17, 339-350.

Bailey, G. (2007). Time perspectives, palimpsests and the archaeology of time. Journal of Anthropological Archaeology, 26(2), 198-223.

Binford, L. (1980). Willow smoke and dog's tails: hunter-gatherer settlement systems and archaeological site formation. American Antiquity, 45, 4-20.

Binford, L. (1981). Bones: Ancient Men and Modern Myths. New York: Academic Press.

Borrazzo, K. (2006). Tafonomía lítica en dunas: Una propuesta para el análisis de los artefactos líticos. Intersecciones en Antropología, 7, 247-261.

Bran, D., Cecchi, G., Balmaceda, A. y Lores, M (1985). Vegetación. En Relevamiento Integrado de los Recursos Naturales de la Provincia de Río Negro, Convenio INTA- Ministerio de Recursos Naturales (Pci de Río Negro). Inédito. Recuperado de Consejo de Ecología y Medio Ambiente (CODEMA), Viedma.

Butzer, K.W. (1982). Geo-arqueología I: Principios Básicos. En Arqueología-una ecología del hombre: Método y teoría para un enfoque contextual (pp 33-40). Barcelona: Ediciones Bellaterra, S.A.

Cabrera A.L. y Willink, A. (1980). Biogeografía de América Latina. Monografías de la OEA, Washington D.C.

Capítoli, R. (1973). El golfo San Matías en el ámbito del mar argentino. En Relevamiento Ecológico y tipificación de las comunidades del Litoral Marítimo de la Provincia de Río Negro, con especial referencia al establecimiento de áreas de cultivo para especies de interés comercial. Instituto de Biología Marina.). pp. 1-10. Asesoría de desarrollo de Río Negro. Consejo Federal de Inversiones. Director del programa: Santiago R. Olivier. Inédito. Recuperado de Biblioteca del Consejo Federal de Inversiones (Capital Federal).

Crisoliti, L.E. y Pahissa Campá, M.E. (1973). Análisis climáticos de las estaciones meteorológicas Patagones, San Antonio Oeste y Trelew. En Relevamiento Ecológico y tipificación de las comunidades del Litoral Marítimo de la Provincia de Río Negro, con especial referencia al establecimiento de áreas de cultivo para especies de interés comercial (pp. 13-88). Instituto de Biología Marina. Asesoría de desarrollo de Río Negro. Consejo Federal de Inversiones. Director del programa: Santiago R. Olivier (Inédito). 
Favier Dubois, C., Borella, F., Manzi, M., Cardillo, M., Lanzellotti, F., Scartascini, M. Carolina y Borges Vaz, E. (2006). Aproximación regional al registro arqueológico de la costa rionegrina. En Cruz, L. y Caracotche, S. (eds.), Arqueología de la Costa Patagónica. Perspectivas para la conservación (pp. 50-68). Río Gallegos, Universidad Nacional de la Patagonia Austral.

Favier Dubois, C. y Borella, F. (2007). Consideraciones acerca de los procesos de formación de concheros de la costa norte del Golfo San Matías, Río Negro. Cazadores Recolectores del Cono Sur, (2), 151-165.

Favier Dubois, C. y Borella, F. (2011). Contrastes en la costa del golfo: Una aproximación al estudio del uso humano del litoral rionegrino en el pasado. En F. Borella y M. Cardillos (comps.), Arqueología de Pescadores y marisqueadores en Nordpatagonia, descifrando un registro de más de 6.000 años (pp. 14-42). Buenos Aires: Editorial Dunken.

Favier Dubois, C., Kokot, R., Scartascini, F y Borella, F. (2016). Una perspectiva geoarqueológica del registro de ocupaciones humanas en el golfo San Matías (Río Negro, Argentina). Intersecciones en Antropología 4, 47-59. Recuperado de https://www.redalyc.org/ pdf/1795/179547946005.pdf

Goldsmith, V. (1985). Coastal dunes. En R.A. Davis Jr (ed.), Coastal sedimentary environments (pp. 303-378). New York: Springer-Verlag.

Klijn, J. (1990). Dune forming factors in a geographical context in Dunes of the European Coasts. Geomorphology-Hydrology-Soils. Catena. Supplement (Giessen), (18), 1-13.

Labraga, J.C. y Villalba, R. (2009). Climate in the Monte Desert: Past trends, present conditions, and future projections. Journal of Arid Environments 73(2), 154-163.

Ley, C., Gallego, J.B. y Vidal, C. (2007). Manual de Restauración de dunas costeras. Dirección General de Costas, Ministerio del Medio Ambiente. Madrid.

Marcos, M.A., Mancini, M. y Favier Dubois, C.M. (2012). Middle-Late Holocene environmental changes in Bajo de la Quinta, NE Patagonia, inferred by palynological records and their relation to human occupation. The Holocene, 256 (11), 1271-1281.

McKee, E.D. (1983). Eolian sand bodies of the world. En M.E. Brookfield y T.S. Ahlbrandt (eds.), Eolian Sediments and proceses (pp. 1-26). Amsterdam: Elsevier.

Quintana, R. (2001). El sitio arqueológico Punta Nagué: Procesos de transformación y estrategias de conservación durante su excavación. Conserva, 5, 39-51.

Roig, F.A., Roig-Juñent, S. y Corbalán, V. (2009). Biogeography of the Monte Desert. Journal of Arid Environment, 73, 164-172.

Sanjaume, E., Gracia, F.J. y Flor, G. (2011). Introducción a la Geomorfología de sistemas dunares. En Sanjaume, E.S., García, P.J. (eds.), Las dunas en España. Sociedad Española de Geomorfología (pp. 10-32). Universidad Gallega, España.

Schäbitz, F. (1994). Holocene climatic variations in northern Patagonia, Argentina. Palaeogeography, Palaeoclimatology, Palaeoecology, 109(2-4), 287-294.

Schäbitz, F. (2003). Estudios polínicos del Cuaternario en las regiones áridas del sur de Argentina. Revista del Museo Argentino de Ciencias Naturales, 5(2), 291-299.

Schellmann, G. y Radtke, U. (2010). Timing and magnitude of Holocene sea-level changes along the middle and south Patagonian Atlantic coast derived from beach ridge systems, litoral terraces and valley-mouth terraces. Earth-Science Reviews, 103, 1-30. 
Schiffer, M. (1983). Toward the identification of formation processes. American Antiquity, 48(4), 673-697.

Schiffer, M.B. (1987). Formation Processes of the Archaeological Record. University of New México Press, Albuquerque.

Tsoar, H. (1990). Trends in the development of sand dunes along the southeastern mediterranean coast in Dunes of the European Coasts. Geomorphology-HydrologySoils. Catena. Supplement (Giessen), (18), 51-60.

Villalba, R. (1994). Tree ring and Glacial Evidence for the Medieval Warm Epoch and the Little Ice Age in Southern South America. Climatic Change, 26, 183-197.

Warren, A. (1979). Eolian Processes. En C. Embleton y J. Thornes (eds.), Processes in Geomophology (pp. 325-351). London: Arnold.

Waters, M.R. (1992). Principles of Geoarchaeology. Tucson \& London: The University of Arizona Press.

Waters, M.R. y Kuehn, D.D. (1996). The geoarchaeology of place: the effect of geological processes on the preservation and interpretation of the archaeological record. American Antiquity, 61(3), 483-497.

\section{Figuras}

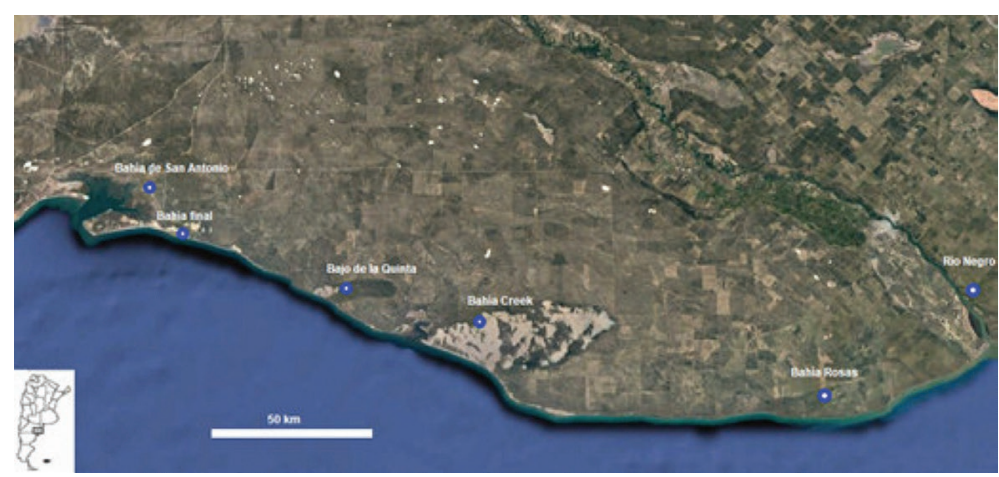

Figura 1. Imagen satelital en la que se destaca la zona de estudio con las localidades que se mencionan en el texto.

Fuente: extraída de Google Earth.

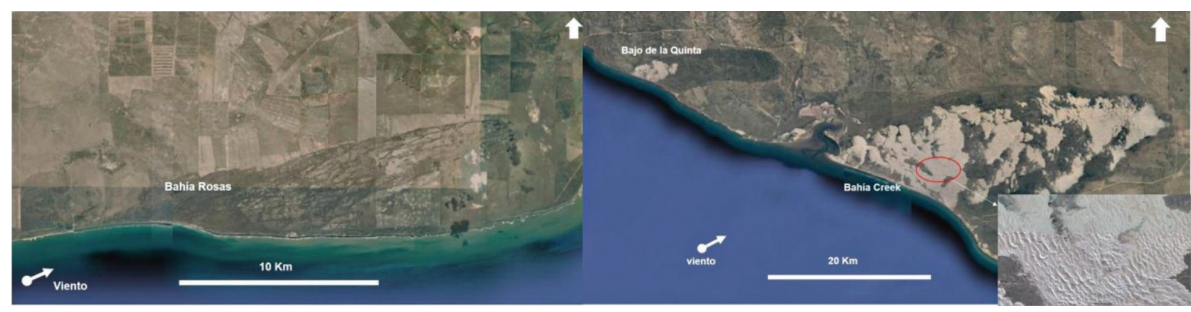

Figura 2. A la Izquierda se destaca la presencia de dunas Parabólicas y a la derecha las dunas Barjanoides, ambas se encuentran en la costa Norte del Golfo de San Matías.

Fuente: extraída de Google Earth. 


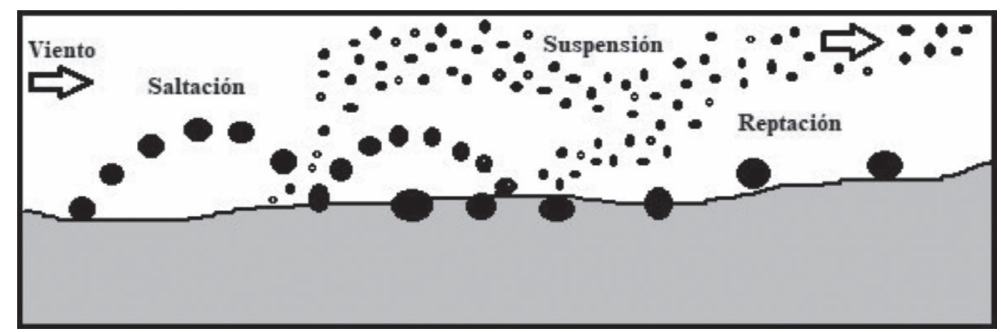

Figura 3. Mecanismos de transporte eólico.

Fuente: de mi autoría.

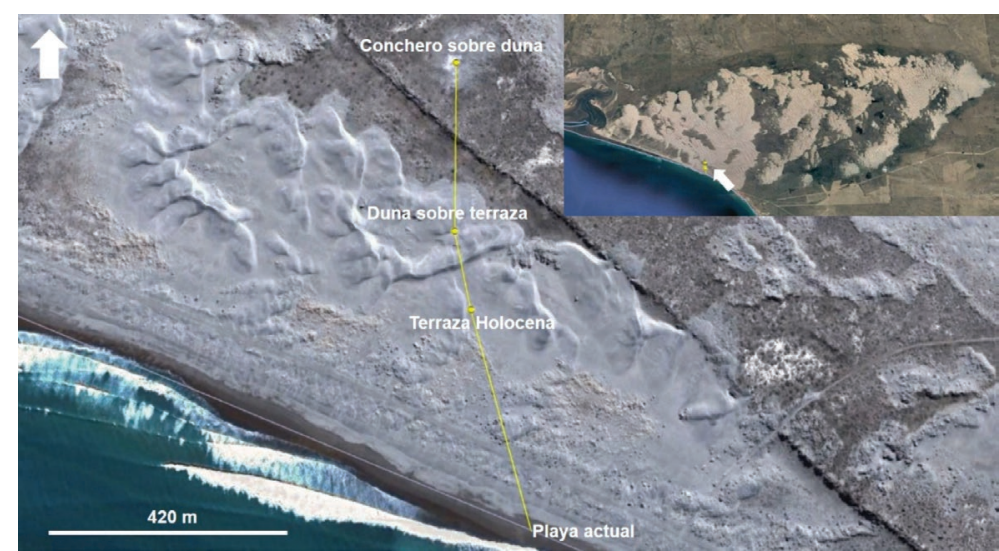

Figura 4. Se detalla en línea amarilla la transecta realizada en las unidades geomorfológicas de Bahía Creek; en el recuadro se destaca con la flecha la ubicación donde se recolectaron las muestras sedimentarias.

Fuente: extraída de Google Earth.

\section{Bahía Creek}

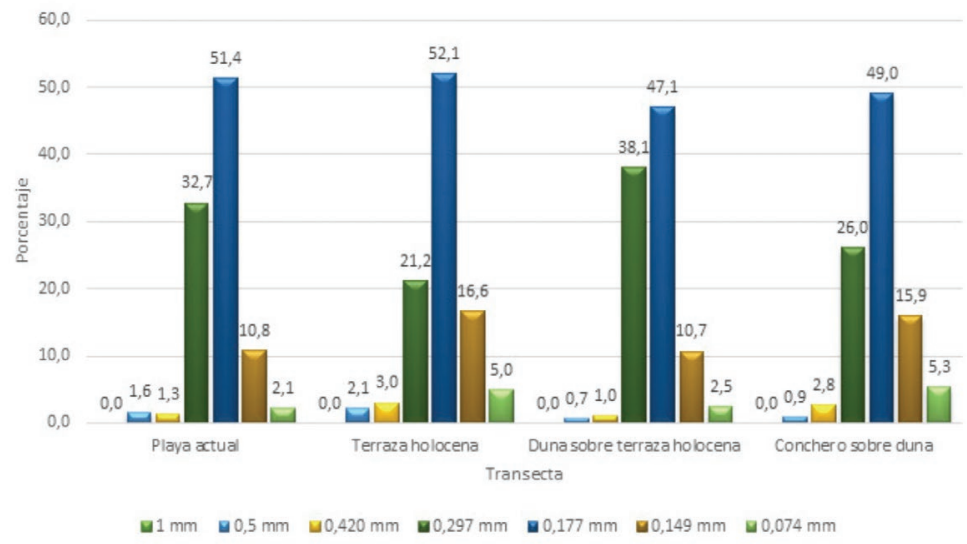

Figura 5. Histograma con porcetaje de tamaño de grano representado en cada unidad geomorfológica muestreada.

Fuente: de mi autoría. 


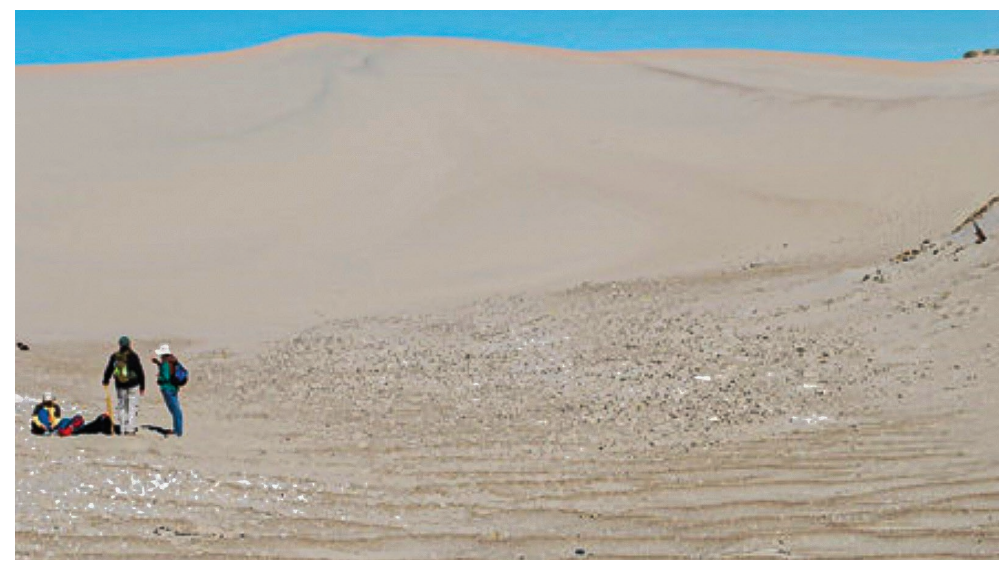

Figura 6. A la izquierda de la imagen exposición de un conchero y a la derecha material expuesto en hoyada de deflación.

Fuente: de mi autoría.

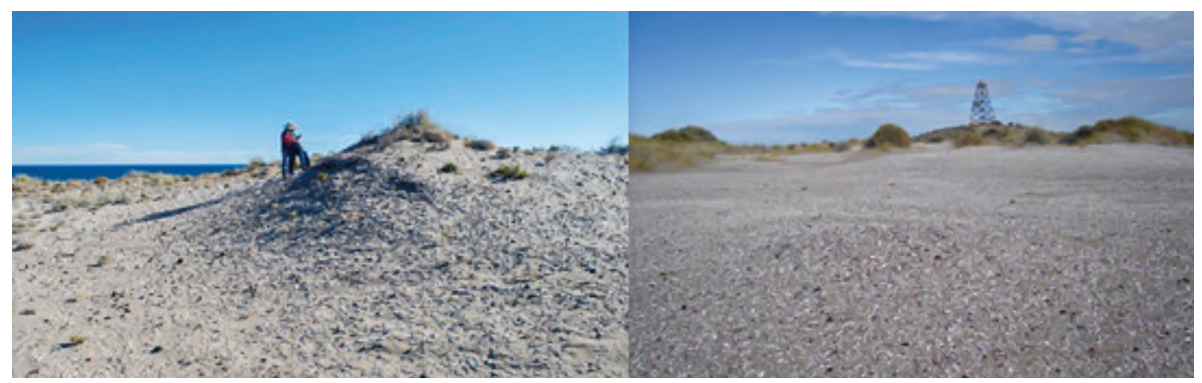

Figura 7. A la izquierda conchero deflacionado y a la derecha morfología derivada de la intensa deflación.

Fuente: de mi autoría.

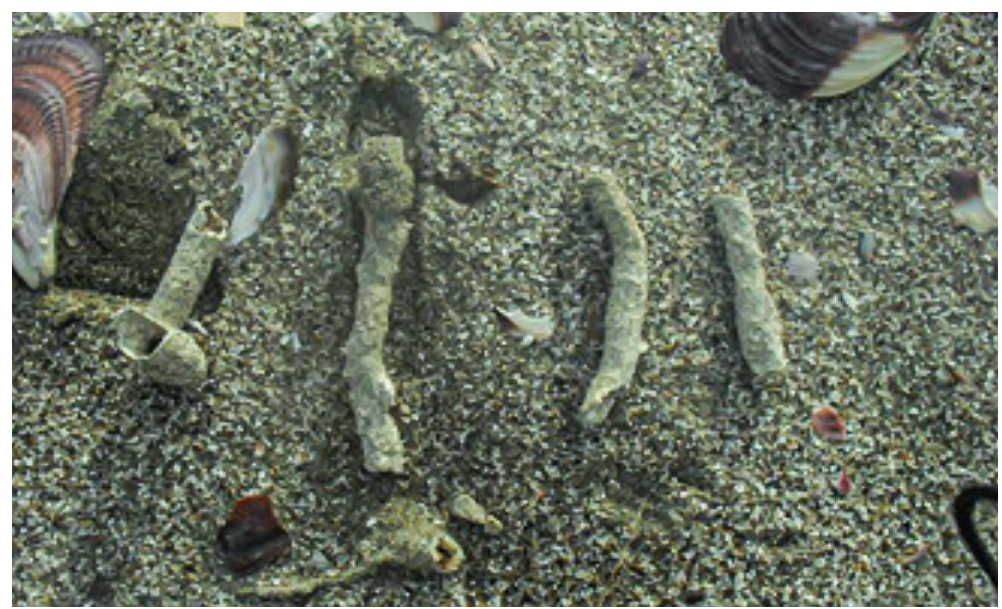

Figura 8. Marcas de raíces-rizoconcreciones.

Fuente: de mi autoría. 